\title{
FOUR-DIMENSIONAL EUCLIDEAN FORMULATION OF QUANTUM FIELD THEORY
}

\section{J. SCHWINGER}

Harvard University, Cambridge (Mass.)
I want to discuss briefly a possible avenue for the future development of quantum field theory, which I believe may be fruitful. We are all accustomed to the idealization that accompanies the quantum theory of fields in its representation of physical phenomena, i.e. the characteristic quantum mechanical feature of the use of abstract vectors and operators to symbolize physical quantities. But in one respect, at least, the quantum field theory has been conservative. It continues to make use of a classical space-time background, upon which the quantum description is superimposed. I would like to suggest a slight deepening of the abstract basis for the representation of physical phenomena, which is the replacement of the Lorentz or Minkowski space by a Euclidean space. At first sight the idea of describing physical phenomena in terms of a Euclidean geometry may appear to be either trivial or incorrect. The aspect of triviality comes from our familiarity with the use of an imaginary time coordinate to bring about an appearance of the Euclidean geometry in the equations of physics. However, this use of an imaginary time co-ordinate is basically absentmindedness, for in classical physics one is very familiar with the fundamental difference between hyperbolic and elliptic differential equations. This already disposes of triviality. It is even less trivial in quantum mechanics because we know that the nature of states is fundamentally related to the underlying symmetry group. That is, we can say that the physical states are, in a sense, representations of the underlying Lorentz group on the one hand, or of the Euclidean group on the other, and these two groups have completely different topologies. This means that while you can certainly take a representation of the Euclidean group and from it derive a representation of the Lorentz group, you will not get all possible representations in this way. What I would like to assert is that while one does not get all the mathematical representations of the Lorentz group, all the representations of physical interest are actually obtained. The essential point to be made is that this possibility of a correspondence between the quantum theory of fields with its underlying Lorentz space, and a mathematical image in a Euclidean space - if one adopts a postulate that one should be able to do this in detail gives results which go beyond what can be obtained from the present theory of fields. These I shall try to indicate.
But besides this, by freeing ourselves from the limitation of the Lorentz group, which has produced all the wellknown difficulties of quantum field theory, one has here a possibility - if this is indeed necessary - of producing new theories. That is, one has the possibility of constructing new theories in the Euclidean space and then translating them back into the Lorentz system to see what they imply. Concerning the second feature, I have done nothing. I am merely suggesting that when one finds formulations that are equivalent, one of these will be distinguished as the one that makes contact with the future theory. All we can do at the moment is to look at all the possible ways of formulating the present theory. First I must demonstrate that there is at least a possibility of replacing the description in Lorentz space by a description in Euclidean space. This depends on making use of suitable objects of correspondence. For this the wellknown vacuum expectation values of time-ordered products are fundamental. These vacuum expectation values, or Green's functions ${ }^{1)}$ as I prefer to call them, are the basic objects which enable us to establish the correspondence between the Lorentz and Euclidean formulations. We consider a general Hermitian field $\chi$, which decomposes into a Bose - Einstein field $\Phi$, and a Fermi - Dirac field $\psi$. The Green's functions can be defined as vacuum state expectation values of time-ordered field operator products. There are two types,

and

$$
G_{+}\left(x_{1} \cdots x_{p}\right)=\left\langle\left(\chi\left(x_{1}\right) \cdots \chi\left(x_{p}\right)\right)_{+}\right\rangle \varepsilon_{+}\left(x_{1} \cdots x_{p}\right)
$$

$$
G_{-}\left(x_{1} \cdots x_{p}\right)=\left\langle\left(\chi\left(x_{p}\right) \cdots \chi\left(x_{1}\right)\right)_{-}\right\rangle \varepsilon_{-}\left(x_{p} \cdots x_{1}\right),
$$

where positive or negative time-ordering implies an assignment of multiplication order in accordance with the ascending sense of time, as read from right to left $(+)$, or from left to right $(-)$. The quantities $\varepsilon_{ \pm}$are antisymmetrical functions of the time coordinates for the F. D. fields, which assume the value +1 when the timeordered sense coincides with the written order. The connection between the two Green's functions is simply

$$
G_{-}\left(x_{1} \cdot x_{p}\right)=G_{+}\left(x_{1} \cdots x_{p}\right) * .
$$

The definitions as given are actually restricted to those 
fields for which all components are kinematically independent at a given time. In more general situations additional terms are necessary ${ }^{1)}$, the function of which is to maintain the non-dependence of the Green's functions on the particular time-like direction employed in the timeordering, which is otherwise assured by the commutativity or anticommutativity of fields at points in space-like relation.

The invariance of the formalism under inhomogeneous Lorentz transformations requires that the Green's functions be translationally invariant functions of the spacetime co-ordinates, while the homogeneous, proper, orthochronous Lorentz transformations

$$
\bar{\chi}(l x)=L \chi(x)
$$

imply the Green's function invariance property

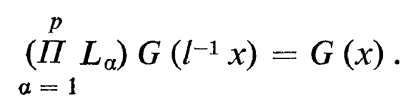

The form of the latter for infinitesimal transformations is

$$
\left[\underset{a=1}{\sum}\left(x_{\mu} \frac{1}{i} \partial_{\nu}-x_{v} \frac{1}{i} \partial_{\mu}+S_{\mu v}\right)_{a}\right] G(x)=0 .
$$

The theory is also invariant under the proper, antiorthochronous transformation $x^{\mu} \rightarrow-x^{\mu}$, provided a transition to the complex conjugate algebra is included ${ }^{2)}$. Now

$$
\begin{aligned}
& {\left[\prod_{\alpha=1}^{p}\left(R_{s t}\right)_{\alpha}\right] G_{+}\left(-x_{1} \cdots-x_{p}\right)^{*}} \\
& =\left\langle\left(R_{s t} \chi^{*}\left(-x_{1}\right) \cdots\right)_{+}\right\rangle \varepsilon_{+}\left(-x_{1} \cdots-x_{p}\right)=G_{-}\left(x_{1} \cdots x_{p}\right),
\end{aligned}
$$

since positive and negative time-orderings are interchanged under time reflection, and, with $n$ pairs of F. D. fields,

$$
\varepsilon_{+}\left(-x_{1} \cdots-x_{p}\right)=(-1)^{n} \varepsilon_{-}\left(x_{p} \cdots x_{1}\right),
$$

while the sign factor $(-1)^{n}$ is compensated by the imaginary unit contained in each matrix $R_{s t}$ that is associated with a half-integral spin (F.D.) field. Thus, for either type of Green's function

$$
\begin{gathered}
{\left[\prod_{\alpha=1}^{p}\left(R_{s t}\right)_{\alpha}\right] G(-x)=G(x)} \\
R_{s t}=e^{\pi i S_{12}} e^{\pi i S_{34}}
\end{gathered}
$$

which, in its union of two disjoint pieces of the Lorentz group, is a sign of the Euclidean foundation on which the Green's functions rest.

The explicit dependence of the fields on the space-time co-ordinates is governed by the energy-momentum vector $P^{\mu}$, according to

$$
\chi(x)=e^{-i P x} \chi e^{i P x},
$$

while the invariant meaning of the vacuum state is expressed by

$$
\left\langle 0\left|e^{-i P x}=\left\langle 0\left|, \quad e^{i P x}\right| 0\right\rangle=\right| 0\right\rangle .
$$

Hence, if $x^{(1)} \cdots x^{(p)}$ represent the time-ordered arrangement of $x_{1} \cdots x_{p}\left(t^{(1)}>\cdots>t^{(p)}\right)$, we have

$$
\begin{aligned}
G_{+}(x)=\left\langle\chi e^{i P\left(x^{(1)}-x^{(2)}\right)} \chi\right. & \cdots \\
& \left.e^{i P\left(x^{(p-1)}-x^{(p)}\right)} \chi\right\rangle \varepsilon_{+}\left(x_{1} \cdots x_{p}\right)
\end{aligned}
$$

and

$$
\begin{aligned}
G_{-}(x)=\left\langle\chi e^{i P\left(x^{(p)}-x(p-1)\right.}\right) & \chi \cdots \\
& \left.e^{i P\left(x^{(2)}-x^{(1)}\right)} \chi\right\rangle \varepsilon_{-}\left(x_{p} \cdots x_{1}\right),
\end{aligned}
$$

wherein additional indices are needed to distinguish the various type of fields. The time dependence of the Green's function $G_{+}$is thus governed by the operators

$$
e^{-i P^{0}\left(t^{(\alpha)}-t^{(\alpha+1)}\right)},
$$

which, in their dependence upon the differences of the consecutively ordered time co-ordinates, contain no negative frequencies $\left(P^{0^{\prime}} \geqslant 0\right)$. The alternative Green's function $G_{-}$, analogously constructed from the operators

$$
e^{i P^{0}\left(t^{(\alpha)}-t^{(\alpha+1)}\right)},
$$

contains no positive frequencies.

We shall now use these spectral characteristics of the Green's functions to give a more precise meaning to the assumed existence of the Green's functions, which is in the sense of the summability of Fourier integrals. It is described by the absolute convergence (for distinct $x_{1} \cdots x_{2}$ ) of the spectral representation obtained on replacing the positive frequency unitary operators in $G_{+}$with

$$
e^{-i P^{0}\left(t^{(\alpha)}-t^{(\alpha+1)}\right)(1-i \varepsilon)}
$$

and similarly inserting

$$
\boldsymbol{e}^{i P^{0}\left(t^{(\alpha)}-t^{(\alpha+1)}\right)(1+i \varepsilon)}
$$

in $G_{-}$, where the limit $\varepsilon \rightarrow+0$ is to be eventually performed. This modified time dependence is also expressed by the substitutions

$$
t_{\alpha} \rightarrow t_{\alpha}(1-i \varepsilon) \text { in } G_{+}, \quad \text { and } \quad t_{\alpha} \rightarrow t_{\alpha}(1+i \varepsilon) \text { in } G_{-} .
$$

The existence of the Green's functions in this sense is equivalently described by the assumption that the various field operator matrix elements, multiplied by the densities of relevant states, possess no more than an algebraic growth with increasing energy.

The absolute convergence of the spectral representations for the Green's functions $G_{+}$and $G_{-}$is now assured for the more general time substitution ${ }^{(*)}$ :

(*) The full analytic extension of the Green's functions $G_{ \pm}$is produced by $t \rightarrow \xi_{\mp} i \eta$ where $\eta$ retains the initial time order, which is to say that the otherwise arbitrary mapping function $\eta(t)$ is of positive slope. 


$$
\begin{array}{rlrl}
G_{+}: & t_{\alpha} \rightarrow \tau_{\alpha} e^{-i \theta} & \sin \theta>0 \\
G_{-}: & t_{\alpha} \rightarrow \tau_{\alpha} e^{i \theta}
\end{array}
$$

in which $\vartheta$ lies in the open interval $0<\vartheta<\pi$. The new variables $\tau_{\alpha}$ are real numbers that retain the ordering of the original time variables. We adopt a special notation to accompany the particular choice $\vartheta=\frac{1}{2} \pi$, which asserts the existence of the functions $G_{+}\left(t \rightarrow-i x_{4}\right)$ and $G_{-}\left(t \rightarrow+i x_{4}\right)$. In this way there emerges a correspondence between the Green's functions in space-time and functions defined on a four-dimensional Euclidean manifold. To the extent that the two Euclidean functions thus obtained are related, there also appears an analytical continuation that connects the two distinct types of spacetime Green's functions, $G_{ \pm}$. Conversely, given one of the Euclidean functions, the substitutions $x_{4} \rightarrow e^{i(\pi / 2-\varepsilon)} t$ and $x_{4} \rightarrow e^{-i(\pi / 2-\varepsilon)} t$ will yield functions having the space-time character of $G_{+}$and $G_{-}$, respectively, in the limit as $\varepsilon \rightarrow+0$. We must now see how to supply an independent basis for the Euclidean Green's functions, from which has disappeared all reference to the space and time distinctions of the Lorentz metric.

The significance of the latter remark can be appreciated through the form assumed in the Euclidean description by the statement of infinitesimal rotational invariance of the Green's functions. The Hermitian spin matrices $S_{\mu \nu}, \mu, \nu=1, \cdots, 4 \quad\left(x_{4}= \pm i x^{0}\right)$, comprising $S_{k l}$ and $S_{k 4}= \pm i S_{0 k}$, still bear the mark of their Lorentz origin in the symmetry of these matrices; the $S_{k l}$ are antisymmetrical, while the $S_{k 4}$ are symmetrical. Hence, one must perform a unitary transformation to unite them into six antisymmetrical, imaginary matrices that describe independent infinitesimal orthogonal transformations. The means for distinguishing between the two types of matrices is provided by the space-reflection matrix $R_{s}$,

$$
R_{s}^{-1} S_{k l} R_{s}=S_{k l}, \quad R_{s}{ }^{-1} S_{k 4} R_{s}=-S_{k 4},
$$

or, alternatively, by the time-reflection matrix

$$
R_{t}=R_{s t} R_{s}
$$

Indeed, for integral spin the necessary transformation is produced by

$$
S_{\mu \nu}(E)=e^{\mp \frac{\pi i}{4} R_{t}} S_{\mu \nu} e^{ \pm \frac{\pi i}{4} R_{t}}
$$

where the plus or minus sign applies to the matrices associated with $G_{+}$or $G_{-}$:

$$
S_{k 4}{ }^{(E)}=e^{\mp \frac{\pi i}{4} R_{t}}( \pm i) S_{0 k} e^{ \pm \frac{\pi i}{4} R_{t}}=R_{t} S_{0 k} .
$$

Since the Hermitian matrices $R_{s}$ and $R_{t}$ are symmetrical ${ }^{3)}$ for integer spin, and anticommute with $S_{0 k}$, the net effect of the transformation is to convert the symmetrical matrix $S_{k 4}$ into the desired antisymmetrical $S_{k 4}{ }^{(E)}$. For halfintegral spin, however, both the skew-Hermitian matrix $R_{S}$ and the Hermitian matrix $R_{t}$ are antisymmetrical and the transformation as stated does not yield the required
Euclidean matrices. One must also associate with $R_{s}$, say, an antisymmetrical matrix that commutes with all $S_{\mu v}$ and with $R_{s}$ (the apparently different possibility of a symmetrical matrix that anticommutes with $R_{s}$, is simply a change of representation ${ }^{3)}$ ), produced with the aid of $R_{s t}$, that effectively replaces $R_{s}$ with $R_{t}$. Thus, to permit the complete transformation from the Lorentz to the Euclidean metric, every half-integer spin (F.D.) field must carry a charge. Just such a general fermionic charge property, under the name of nucleonic charge or leptonic charge, is either well established experimentally, or has been conjectured on other grounds. The Euclidean formulation may be the proper basis for comprehending this general attribute of F. D. fields. If $\ell$ is the imaginary antisymmetrical matrix representing the fermionic charge property, the required transformation for half-integer spin fields is

$$
S_{\mu \nu}(E)=e^{ \pm \frac{\pi}{4} R_{S} \ell} S_{\mu \nu} e^{\mp \frac{\pi}{4} R_{S} \ell}
$$

and, indeed,

$$
S_{k 4}{ }^{(E)}=i \ell R_{s} S_{v k}
$$

has the desired property of antisymmetry.

We should also note the removal of reference to the Lorentz metric from the orthogonal matrices $R_{k}, R_{t}$, that are associated with the reflections of the individual spacetime co-ordinate axes. For a B.E. field all these matrices are commutative, real, symmetrical matrices, and they are unchanged by the transformation to the Euclidean metric. On considering a F.D. field, however, we find that $R_{t}$, occupies a distinguished position, differing from the anticommuting, real, symmetrical matrices $R_{k}$ by being imaginary and antisymmetrical. While the matrices $R_{k}$ are unaltered by the metric change, the Euclidean matrix associated with the reflection of $x_{4}$ is now

$$
R_{4}(E)=e^{ \pm \frac{\pi}{4} R_{S} \ell}\left( \pm R_{t}\right) e^{\mp \frac{\pi}{4} R_{S} \ell}=R_{s t} \ell,
$$

which is also a real symmetrical matrix. Thus all the individual Euclidean co-ordinate reflection matrices are real, symmetrical and orthogonal, with the two classes of fields distinguished by commutativity properties, according to

$$
E: \quad R_{\mu} R_{\nu}=e^{\pi i S_{\mu \nu}}=e^{2 \pi i S_{\mu \nu}} R_{\nu} R_{\mu} .
$$

To obtain an independent characterization of the Euclidean-type Green's functions we can convert to the Euclidean metric the system of differential equations obeyed by the Green's functions. We shall only attempt to outline this process in the following considerations. Let the Lagrange function be written as

$$
\mathfrak{L}=\frac{1}{4}\left[\chi A^{\mu} \partial_{\mu} \chi-\partial_{\mu} \chi A^{\mu} \chi\right]+\frac{1}{2} \chi B \chi-\mathfrak{S}_{1}(\chi),
$$

in which $\mathfrak{H}_{1}$ refers to the interactions between fields. The field equations are

$$
A^{\mu} \partial_{\mu} \chi+B \chi=\frac{\partial_{l} \mathfrak{H}_{1}}{\partial \chi}
$$


while the commutation properties on a space-like surface, as expressed in a local co-ordinate system, are given by (for simplicity of description, we adhere to the fiction that all components of $\chi$ are kinematically independent on $\sigma)$

$$
\left[A^{0} \chi(x), \chi\left(x^{\prime}\right)\right]_{ \pm}=i \delta^{0}\left(x-x^{\prime}\right),
$$

where $\delta^{\mu}\left(x-x^{\prime}\right)$ is defined by

$$
\int d \sigma_{\mu}^{\prime} \delta^{\mu}\left(x-x^{\prime}\right) f\left(x^{\prime}\right)=f(x) .
$$

Now, uniting the field equations and commutation properties, the differential equations for the Green's functions are obtained :

$$
\begin{aligned}
& \left(A^{\mu} \partial_{\mu}+B\right)_{1} G_{+}\left(x_{1} \cdots x_{p}\right)+\ldots \\
& =i \delta\left(x_{1}-x_{2}\right) G_{+}\left(x_{3} \cdots x_{p}\right) \pm i \delta\left(x_{1}-x_{3}\right) G_{+}\left(x_{2} \cdots x_{p}\right)+\ldots
\end{aligned}
$$

in which the omitted terms on the left are the particular Green's function combinations needed to represent the interaction effects in the field equations, while, on the right, the summation is extended over all points that refer to the same field as does $x_{1}$. The summation is symmetrical or antisymmetrical in these points, according to the statistics of the field. Thus the Green's functions obey an infinite system of equations that are linear and inhomogeneous (since the function with $p=0$ is simply unity), and which incorporate fully all information concerning the interacting fields.

The analogous differential equations for the $G_{-}$can be obtained directly, or by complex conjugation of the equations obeyed by $G_{+}$and differ from the latter only in the sign of $i$ exhibited by the right-hand $\mathrm{B}$. E. terms. It is worthy of note that, apart from the trivial situation of uncoupled fields, the two sets of differential equations are intrinsically different - the two types of Lorentztype Green's functions cannot be characterized in detail as solutions of a common equation that are distinguished by boundary conditions. There is, however, a simple relation between the Green's functions that can be inferred from the differential equations (and from the time ordered operator definitions), namely

$$
G_{-}(x)=(-1)^{n} G_{+}\left(-e^{-\pi i} x\right) .
$$

The factor $(-1)^{n}$ is inserted to reverse the sign of the righthand F.D. terms. The interpretation given to $-e^{-\pi i}$, applied to the space coordinates, is unity. But, for the time co-ordinates, it is a combination of two operations : time reflection, which inverts the time order $\left(t^{(1)}>\cdots t^{(p)}\right.$, $\left.-t^{(p)}>\cdots-t^{(1)}\right)$; multiplication by $e^{-\pi i}$, which reverses the sign of all time coordinates while retaining the time order. The latter transformation changes the sign of all the $\delta$-functions and converts the differential equations for $G_{+}$into those describing $G_{-}$. The negative frequency character of the latter functions is also reproduced. The connection here obtained between $G_{+}$and $G_{-}$is an analytic continuation stated without reference to intermediate Euclidean functions.
The correspondence between Lorentz- and Euclideantype Green's functions is now exhibited as

$$
\begin{aligned}
G_{ \pm}(x) \longleftrightarrow & \Pi_{B . E .}\left(e^{ \pm \frac{\pi i}{4} R t} e^{\mp^{\pi i}}\right)_{\alpha} \\
& \times \Pi_{F . D .}\left(e^{\mp \frac{\pi}{4} R_{S} \ell} e^{\mp \frac{\pi i}{4}}\right)_{\alpha} G_{ \pm}(x)^{(E)},
\end{aligned}
$$

in which $x^{0}$ and $x_{4}$ are understood as the variables in the appropriate functions. Accompanying the imaginary relations between the variables is the transformation

$$
\delta(x) \leftarrow( \pm i) \delta(x)^{(E)} .
$$

On performing these substitutions in the differential equations we encounter the real B.E. matrices

$$
\begin{aligned}
& A_{\mu}^{(E)}=-e^{ \pm \frac{\pi i}{4} R_{t}} e^{\mp \frac{\pi i}{4}} A_{\mu} e^{ \pm \frac{\pi i}{4} R_{t}} e^{\mp \frac{\pi i}{4}} \\
& =\left(-A_{k} R_{t}, A_{0}\right) \\
& \text { B.E. : } \quad B^{(E)}=-e^{ \pm \frac{\pi i}{4} R t} e^{\mp \frac{\pi i}{4}} B \quad e^{ \pm \frac{\pi i}{4} R_{t}} e^{\mp \frac{\pi i}{4}} \\
& =-B R_{t},
\end{aligned}
$$

which are, respectively, antisymmetrical and symmetrical, and the real F.D. matrices

$$
A_{\mu}^{(E)}=\mp e^{\mp \frac{\pi}{4} R_{S} \ell} e^{\mp \frac{\pi i}{4}} A_{\mu} e^{\mp \frac{\pi}{4} R_{S} \ell} e^{\mp \frac{\pi i}{4}}=
$$

F.D. :

$$
\begin{aligned}
B^{(E)} \ell=-e^{\mp \frac{\pi}{4} R_{s} \ell} e^{\mp \frac{\pi l}{4}} B e^{\mp \frac{\pi}{4} R_{s} \ell} e^{\mp \frac{\pi i}{4}} & = \\
& -B R_{s} i \ell,
\end{aligned}
$$

which are symmetrical and antisymmetrical, respectively. The resulting form of the Green's function differential equations, as adapted to the Euclidean metric, is

$$
\begin{aligned}
{\left[A_{\mu} \partial_{\mu}+(1, \pm \ell) B\right]_{1} } & G_{ \pm}\left(x_{1} \cdots x_{p}\right)^{(E)}+\ldots \\
= & \delta\left(x_{1}-x_{2}\right) G_{ \pm}\left(x_{3} \cdots x_{p}\right)^{(E)}+\ldots
\end{aligned}
$$

where the choice indicated for the coefficient of $B$ signifies : unity, for a B.E. field; $\pm \ell$, for a F.D. field. If, as we have discussed before ${ }^{3)}$, the Lorentz $B$ matrices are constructed as the product of $R_{s}$ with an invariant, symmetrical matrix that is independent of internal degrees of freedom, the Euclidean $B$ matrices will be completely of the latter type. This remark, together with the observation that the transformation applied to $B^{-1} A_{\mu}$ is unitary, apart from the F.D. factor $\pm \ell$, indicates how Lorentz invariance is translated into Euclidean invariance.

The Green's functions of the Lorentz description are intrinsically complex quantities and, accordingly, there are two linearly independent sets of such functions. It is an indication of the simplification obtained through the introduction of the Euclidean metric that completely real Euclidean Green's functions can be defined - provided a certain general symmetry restriction is enforced on the field interactions. Conversely, the latter invariance property acquires substantial support through its role in 
unifying the two classes of Green's functions and eliminating complex numbers from a formulation of the fundamental laws of physics. Let us notice that, aside from the interaction terms, the differential equations for $G_{ \pm}(E)$ differ only in the F.D. quantity $\pm \ell$, referring to the fermionic charge. This sign factor can be removed by introducing the operation of fermionic charge reflection. But, when full account is taken of the variety of field interactions ${ }^{4)}$ it appears that all types of charge are dynamically coupled, and the interconversion of the two sets of equations is possible only if the interaction terms differ merely through the effect of general charge reflection. Assuming this property, we conclude that

$$
G_{-}{ }^{(E)}=\left[\underset{a=1}{p}\left(R_{Q}\right)_{\alpha}\right] G_{+}{ }^{(E)}=R_{Q} G_{+}{ }^{(E)},
$$

where the individual charge reflection matrices $R_{Q}$ are real and orthogonal. The composite matrix $R_{Q}$ also describes the reality properties of the Euclidean-type Green's functions,

$$
G_{+}(E) *=R_{Q} G_{+}^{(E)},
$$

for the mutually complex conjugate relation of $G_{ \pm}$still applies to the derived functions $G_{ \pm}{ }^{(E)}$. In effect, all matrices appearing in the Euclidean formulation of the differential equations are real, with the exception of the imaginary charge matrices, and complex conjugation is equivalent to charge reflection. If we accept the interpretation ${ }^{2)}$ of the imaginary unit as symbolic of the charge nature of the measurement apparatus (matter - antimatter), the symmetry property we have postulated can be described as the relativistic invariance of the Euclidean formulation with respect to charge reflection, for the application of this transformation to the system under investigation and to the apparatus employed for the purpose produces no discernible change.

Before continuing, we must examine the relation between the matrices $R_{Q}{ }^{(E)}$, and the charge reflection matrices of the Lorentz description, $R_{Q}$. The latter, having no reference to space-time properties, are uniformly chosen as real, orthogonal, symmetrical matrices $\left(R_{Q}{ }^{2}=1\right)$. The distinction between $R_{Q}$ and $R_{Q}{ }^{(E)}$, which exists only for F.D. fields, arises from the incorporation of the fermionic charge $\ell$ into $A_{4}{ }^{(E)}=-A_{o} R_{S} \ell$. To compensate the sign change of $A_{4}{ }^{(E)}$ induced by the reflection of $\ell$, the F.D. matrix $R_{Q}{ }^{(E)}$ must contain the co-ordinate reflection matrix $R_{4}{ }^{(E)}=R_{s t} \ell$. Thus,

$$
\text { F.D. : } \quad R_{Q}^{(E)}=R_{Q} R_{s t} \ell,
$$

which is a real, orthogonal, antisymmetrical matrix $\left(R_{Q}{ }^{(E) 2}=-1\right)$. However, the composite matrix $R_{Q}{ }^{(E)}$, which is constructed from an even number of F. D. contributions, is a real symmetrical matrix obeying

$$
R_{Q^{(E) 2}}=1
$$

The hypothesis of Euclidean relativistic charge reflection invariance can now be interpreted as a property of the Lorentz-type Green's functions and, thereby, of the Lagrange function of the interacting fields. The relation implied between the Green's functions $G_{+}$and $G_{-}$is

$$
\begin{aligned}
G_{-}\left(i x_{4}\right) & =\Pi_{B . E,}\left(R_{Q} R_{t}\right) \Pi_{F . D .}\left(R_{Q} i R_{t}\right) G_{+}\left(-i x_{4}\right) \\
& =(-1)^{n} R_{Q} R_{t} G_{+}\left(-i x_{4}\right)
\end{aligned}
$$

which makes explicit the analytic continuation that connects the two Lorentz Green's functions

$$
\begin{aligned}
& G_{-}(t)=(-1)^{n} R_{Q} R_{t} G_{+}\left(e^{-\pi i} t\right) \\
& G_{+}(t)=(-1)^{n} R_{Q} R_{t} G_{-}\left(e^{\pi i} t\right) .
\end{aligned}
$$

When this result is compared with the previously obtained connection,

$$
G_{\mp}(t)=(-1)^{n} G_{ \pm}\left(-e^{\mp \pi i} t\right),
$$

we learn that

$$
R_{Q} R_{t} G(-t)=G(t)
$$

the Lorentz-type Green's functions are invariant under charge and time reflection. The same assertion can be made of the combination of charge and space reflection, since space-time reflection is an invariance operation. But in the latter form we are dealing with unitary transformations of Hermitian field operators, and it can be concluded that the invariance of the Lagrange function under space and charge reflection is equivalent to the postulate that Euclidean-type Green's functions exhibit a relativistic invariance with respect to charge reflection. It is surely significant that we are thus led to a general invariance property which is consistent with all the recent experiments on the so-called parity non-conserving interactions. The existence of an exact invariance transformation involving space reflection is now supplied with a basis that may be considered more substantial than the mere belief in the intrinsic indiscernibility of left and right.

We have not yet exhibited the real Euclidean-type Green's functions, the existence of which is assured by the presence of a linear transformation equivalent to complex conjugation,

$$
\left(G_{ \pm}(E)\right) *=R_{Q} G_{ \pm}^{(E)}
$$

Indeed, functions having the required reality property are given by

$$
G^{(E)}=e^{\frac{\pi i}{4} R_{Q}} e^{-\frac{\pi i}{4}} G_{+}{ }^{(E)}=e^{-\frac{\pi i}{4} R_{Q}} e^{\frac{\pi i}{4}} G_{-}{ }^{(E)} .
$$

A second such choice is

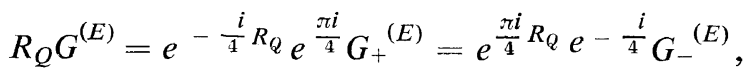

although the latter are not a linearly independent set and can be regarded as presenting $G^{(E)}$ in a new representation. 
An essential limitation of the description by real Green's functions must be observed, however. The matrices $e^{ \pm \frac{\pi i}{4} R_{Q}}$ are not composite and the transformation that introduces $G^{(E)}$ has no simple significance for the differential equations that characterize the Green's functions. Were only B.E. fields involved, the composite transformation formed from the individual $R_{Q}$ could be employed, but this is not possible for F.D. fields. Nevertheless, it remains true that real Euclidean-type Green's functions exist from which the physically meaningful Green's functions $G_{+}$and $G_{-}$can be inferred.

Finally, we shall indicate briefly the possibility of replacing the differential equations, as the characterization of the Euclidean-type Green's functions, by an explicit if formal construction. For this purpose we define fields $\chi(x)$, on the Euclidean manifold, that are completely commutative or anticommutative, as befits the statistics,

$$
\left[\chi(x), \chi\left(x^{\prime}\right)\right]_{ \pm}=0
$$

and a complementary set of fields $\hat{\chi}(x)$, with the same characteristics, which are such that

$$
\left[\hat{\chi}(x), \chi\left(x^{\prime}\right)\right]_{ \pm}=\delta\left(x-x^{\prime}\right) .
$$

The B.E. fields $\Phi, i \hat{\Phi}$ are Hermitian, while the F.D. fields $\psi$ and $\hat{\psi}$ are mutually Hermitian conjugate. The Euclidean-type Green's functions are then given by

$$
G_{ \pm}\left(x_{1} \cdots x_{p}\right)^{(E)}=\left\langle W_{ \pm}\left|\chi\left(x_{1}\right) \cdots \chi\left(x_{p}\right)\right| 0\right\rangle /\left\langle W_{ \pm} \mid 0\right\rangle,
$$

(ordinary operator multiplication!) where $|0\rangle$ is the right eigenvector of the operators $\hat{\chi}(x)$ associated with null eigenvalues

$$
\hat{\chi}(x)|0\rangle=0
$$

and the vector $\left\langle W_{ \pm}\right|$is characterized by

$$
\left\langle W_{ \pm}\right|\left[A_{\mu} \partial_{\mu} \chi+(1, \pm \ell) B \chi+\ldots-\hat{\chi}\right]=0,
$$

in which the omitted terms are the functions of the Euclidean field operators $\chi(x)$ needed to describe the field interactions. This assertion is verified on observing that

$$
\begin{aligned}
{\left[A_{\mu} \partial_{\mu}+(1, \pm \ell) B\right]_{1} G_{ \pm}\left(x_{1} \cdots x_{p}\right)^{(E)}+\ldots } \\
\quad=\left\langle W_{ \pm}\left|\hat{\chi}\left(x_{1}\right) \chi\left(x_{2}\right) \cdots \chi\left(x_{p}\right)\right| 0\right\rangle /\left\langle W_{ \pm} \mid 0\right\rangle \\
\quad=\delta\left(x_{1}-x_{2}\right) G_{ \pm}\left(x_{3} \cdots x_{p}\right)^{(E)}+\ldots,
\end{aligned}
$$

where commutation relations and the significance of $|0\rangle$ as a $\hat{\chi}$ eigenvector are used to obtain the stated result.

The vector $\left\langle W_{ \pm}\right|$can be constructed from the left eigenvector of the $\hat{\chi}$ associated with null eigenvalues,

$$
\left\langle W_{ \pm}\right|=\langle 0| e^{-W_{ \pm}}
$$

where the operator

$$
W_{ \pm}[\chi]=\frac{1}{2} \int(d x)\left[\chi A_{\mu} \partial_{\mu} \chi+\chi(1, \pm \ell) B \chi+\ldots\right]
$$

bears an obvious genetic relation to the action operator of the interacting fields. The Euclidean operators $W$ are not Hermitian but they are related to their Hermitian conjugates by a unitary transformation, which constitues a selfadjointness property. We now have

$G_{ \pm}(x)^{(E)}=\left\langle 0\left|\chi\left(x_{1}\right) \cdots \chi\left(x_{p}\right) e^{-W_{ \pm}[\chi]}\right| 0\right\rangle \mid\left\langle 0\left|e^{-W_{ \pm}}\right| 0\right\rangle$

and, in turn, these Green's functions can be derived from a single generating function, the expansion of which produces the field operator products. In the latter form, we make contact with previous developments employing the action principle for quantized fields and the device of external sources ${ }^{1)}$ and subsequent work, largely unpublished). A large variety of equivalent forms can now be devised for the Green's functions, based primarily upon the well-established transformation and representation theory $\left.{ }^{*}\right)$ for canonical variables of the first and second kind. A discussion of these developments for specific systems will be deferred to another publication, in which the problem of translating quantum electrodynamics into the Euclidean metric is examined.

Although we have emphasized the fundamental implications of the Euclidean representation, it will be evident that the Euclidean-type Green's functions also have practical advantages. Indeed, the utility of introducing a Euclidean metric has frequently been noticed in connection with various specific problems, but an appreciation of the complete generality of the procedure has been lacking.

\section{LIST OF REFERENCES}

1. Schwinger, J. Proc. Nat. Acad. Sci. USA., 37, p. 452, 1951

2. Schwinger, J. Proc. Nat. Acad. Sci. USA., 44, p. 223, 1958.

Jost, R. Helv, phys. Acta, 30, p. 409, 1957.

3. Schwinger, J. Proc. Nat. Acad. Sci. USA., 44, p. 617, 1958.

4. Schwinger, J. Ann. Phys. New-York, 2, p. 407, 1957.

5. Schwinger, J. Quantum theory of fields, in: Handbuch der Physik; volume V/2, Berlin, Springer (to be published)

(*) An extended disçussion is contained in ${ }^{5}$. 


\section{DISCUSSION}

Chairman: I thank you very much for this inspiring report. To open the discussion I wish to say that for the audience it is perhaps a bit more interesting than for the speaker that the idea of analytical continuation has been anticipated by Wightman. Instead of more general transformations the speaker has selected a particular case of rotations of $90^{\circ}$, and I hope I interpret him correctly that he means that this has a special significance for physics and for the formalism in that particular case.

Yamaguchi: I just want to add a brief comment on this paper. Nakano of Osaka City University tried the same proposal and he is busy working on this proposal. That is all.

Stueckelberg: I would like to tell Schwinger that if you take a real Hilbert space, and $i$ as an antisymmetric operator, unitary meaning now orthogonal, in which all observables are symmetric observables, you must take $i$ commuting with all observables, $i^{2}=-1$, meaning just that $i$ has an inverse, and you get the $C T P$ invariance stated in a real linear way. Pauli tells me that this is a triviality and probably it is. Now if you take an indefinite metric, does the spin statistics relation, usually a consequence of the positive definite energy - that is, of causality - now follow exclusively from the $T C P$ invariance in this formalism?
Pauli: (to Schwinger) Perhaps you can see in general how the spin statistics connection comes out in this Euclidean interpretation. This would be interesting.

Schwinger: First, the $T C P$ theorem is built into the theory since the fundamental way in which one understands it is through the underlying Euclidean formulation of the theory. That is, in the ordinary description one produces the time reversal essentially by going outside the Lorentz group through the Euclidean group or the complex Lorentz group, which is even more general, as Jost has done, in order to produce the $T C P$ transformation. The $T C P$ theorem is one of the indications for the underlying Euclidean structure of the present-day theory.

When one has presented the theory in a Euclidean form it is automatically contained, as there is no longer any distinction between the past and the future, and the proper transformation, which includes time reflection, is a consequence of the ordinary rotations of the theory. This, of course, depends upon the spin statistics connection and the whole development has already made use of it. The question of to what extent you can go backwards, remains unanswered, i.e. if one begins with an arbitrary Euclidean theory and one asks : when do you get a sensible Lorentz theory? This I do not know. The development has been in one direction only; the possibility of future progress comes from the examination of the reverse direction, and that is completely open. 